\title{
Las casas prefabricadas de las fincas cafetaleras del Soconusco, México.
}

\author{
Ana Johari Mejia ${ }^{1}$ \\ Universidad Nacional Autónoma de México
}

\section{RESUMEN:}

Las casas prefabricadas construidas por empresas de EUA, entre finales del siglo XIX y principios del XX, llegaron al Soconusco mexicano (en el estado de Chiapas) con la migración de familias germano-guatemaltecas para constituir fincas dedicadas a la producción de café lavado destinado a la exportación mundial. Este texto, indagará sobre el origen de las casas prefabricadas y buscará dar explicación sobre los motivos de adquisición, a la vez que documentará dos casas construidas a partir de este método de prefabricación de elementos y posterior ensamblaje en obra.

PALABRAS CLAVES:

casas prefabricadas, fincas de café, arquitectura, Soconusco, México.

\section{ABSTRACT:}

The prefabricated houses built by companies in the USA, between the late nineteenth and early twentieth centuries, reached the Mexican Soconusco (located in the state of Chiapas) with the migration of German-Guatemalan families who had come to establish coffee plantations dedicated to the production of washed coffee destined for world export. This text investigates the origin of these prefabricated houses, the reasons for their acquisition, while at the same time documenting construction of two houses built by this method and subsequent assembly on site.

\section{KEYWORDS:}

prefabricated houses, coffee plantations, architecture, Soconusco, México.

Becaria del Programa de Becas Posdoctorales UNAM, en el Centro de Investigaciones Multidisciplinarias sobre Chiapas y la Frontera Sur (CIMSUR). Asesorada por el Dr. Fennez. 


\section{Introducción}

En el Soconusco mexicano se conservan algunas edificaciones singulares realizadas en madera, muy coloridas, con grandes porches, que caracterizan a las fincas de café y que se valieron de un sistema constructivo en el que todos sus elementos se prefabricaron y posteriormente se ensamblaron en obra. Estas arquitecturas fueron adquiridas por familias germano-guatemaltecas que colonizaron la región, entre finales del siglo XIX y principios del siglo XX, y que fundaron fincas dedicadas a la producción de café lavado destinado al mercado mundial.

Con la llegada de estas familias se importaron los "buenos estilos" de residir en una casa grande, destinada al disfrute del patrón y de su familia y adquiridas por catálogo a Estados Unidos. En la actualidad estas edificaciones constituyen ejemplos representativos de este tipo de arquitectura en el Sureste Mexicano y son vestigios del patrimonio arquitectónico e industrial de las fincas cafetaleras.

Muchas de estas casas grandes y demás elementos asociados a la industria del café se han perdido o están en un estado técnico desfavorable, por envejecimiento, abandono o falta de mantenimiento, de modo que es preciso abordar su estudio para profundizar sobre la historia de estas construcciones y revalorizar el legado productivo que ha contribuido al desarrollo económico de la región.

Todos estos argumentos motivaron la investigación que se plantea identificar las arquitecturas prefabricadas de importación vinculadas a las fincas de café con una cronología aproximada de 1890 a 1930. El propósito de este texto es hacer un acercamiento específico de dos viviendas vinculadas a la casa grande de la familia Giesemann, sus señas de identidad (tomando en consideración los modelos que se promocionaban en los catálogos de casas prefabricadas de Estados Unidos), el origen y su cronología. Se busca contribuir en el conocimiento del patrimonio arquitectónico y garantizar su preservación como legado cultural del periodo histórico estudiado.

\section{El sistema constructivo prefabricado}

El sistema prefabricado para las edificaciones de madera fue un sistema muy popular en Estados Unidos a finales del siglo XIX y principios del XX, aunque la historia de la prefa- bricación es anterior y se halla en los esfuerzos colonizadores de Gran Bretaña y la necesidad de proveer a los pioneros ingleses de un refugio que podía ser transportado y erigido de manera rápida y fácil. ${ }^{1}$

La prefabricación se caracteriza porque todos los componentes son manufacturados de principio a fin en una fábrica, usualmente en un lugar con una sofisticada economía, abundante mano de obra y avanzadas técnicas industriales; para posteriormente ser trasportados y ensamblados con el mínimo de mano de obra y habilidades requeridas. ${ }^{2}$ Además, se debe entender que existen varios grados de prefabricación que han ido evolucionando y cambiando con el tiempo, el sistema pre-cortado es uno de ellos, la fabricación de paneles es otro, la construcción de un volumen insertado en una sección es otro y el sistema de manufactura de una construcción entera de una casa móvil es el último y el más reciente. ${ }^{3} \mathrm{~A}$ finales del siglo XIX los sistemas más populares de construcción prefabricada en Estados Unidos fueron los sistemas portátiles y por secciones, precursores del sistema pre-cortado, éste último se vendió a gran escala y se hizo popular con los catálogos de compañías de casas que se compraban por correo postal.

Los primeros precedentes de viviendas prefabricadas datan del XVII, cuando Inglaterra ${ }^{4}$ envió a Estados Unidos una flota pesquera y edificaciones construidas con paneles de madera como residencia doméstica de los trabajadores. ${ }^{5}$ Con ellos se buscaba reducir significativamente la labor en obra para construir de manera estructural y con precisión en ciertos aspectos. Aunque los edificios no se entendían como la prefabricación de hoy en día, sí que

\footnotetext{
ARIEFF, Allison y BURKHART, Bryan, Prefab, Gibbs Smith, Utah, 2002, p. 13.

2 BELL, Peter, "Continuity in Australian Timber Domestic Building: An Early Cottage at Burra”, en Australian Journal of Historical Archeology, Vol. 8, 1990, p. 3.

3 BURHAM, Kelly, "The prefabrication of Houses", en The Technology Press of the Massachusetts Institute of Technology and Wiley, 7, Cambridge, Mass. y Nueva York, 1951, p. 7.

4 Ubicadas en la península de Cape Ann, en lo que ahora es Massachussets.

5 BIGNELL, Marty, "Some Assembly Required: Component and Ensemble in Prefabricated Australian Domestic Construction", en Society of Arquitectural Historians, Australia and New Zeland: 31, Translations, editado por Christoph Schnoor, Auckland, Nueva Zelanda: SAHANZ y Unitec ePress y Gold Coast, Queensland: SAHANZ, 2014, p. 426.
} 
intentaron acercarse al concepto esencial, un concepto de coordinación dimensional y estandarización. ${ }^{6}$

Posteriormente, durante el siglo XVIII, se hizo extensivo el envío de edificios prefabricados a las colonias inglesas del mundo; se enviaron hospitales, escuelas, tiendas o casas pequeñas para la vida en el campo. El efecto económico de este tipo de construcciones fue transferir la demanda de mano de obra de un lugar donde es costoso a un lugar donde es barato. Fue ideal para la economía colonial, con edificios prefabricados suministrados desde la metrópoli a las colonias, o desde las colonias más avanzadas a las colonias menos avanzadas. ${ }^{7}$

Un experimento notable fue el Portable Colonial Cottage, construido en Australia en 1833 por el carpintero inglés John Manning. Su estructura se armaba con pilares de carga espaciados y paneles estandarizados para rellenar las paredes, ensamblando el suelo, los paneles del techo y las vigas de unión con tornillos. El resultado fue una estructura que podía ensamblarse con poco más que una llave de tuercas y que no requería cortes ni uniones de los elementos estandarizados. ${ }^{8}$ La cabaña que Manning había desarrollado era un sistema estructural que presagiaba la arquitectura modernista posterior: producción en masa, montaje por mano de obra no cualificada y estandarización de los componentes de construcción. ${ }^{9}$

A partir de 1830 en Estados Unidos, se hizo un avance significativo del sistema prefabricado, cuando se popularizó el sistema balloon frame, basado en un marco de madera y cuyo origen es un antiguo método de interconexión de maderas pesadas que armaban un esqueleto. ${ }^{10}$ La estructura de madera era construida por carpinteros itinerantes y articulaba tablas juntas puestas de manera cuidadosa. Este método requería un ordenamiento y señalización de las

6 HERBERT, Gilbert, Pioneers o Prefabrication: the British contribution in the 19th century, The John's Hopkins University Press, Baltimore, 1978, pp. 11-12 HERBERT, Gilbert, Pioneers o Prefabrication: the British contribution in the 19th century, opus cit., p. 7

8 HERBERT, Gilbert, Pioneers o Prefabrication: the British contribution in the 19th century, opus cit., p. 7 9 BIGNELL, Marty, "Some Assembly Required: Component and Ensemble in Prefabricated Australian Domestic Construction", opus cit., p. 427.

10 JOHNSON, Rachel, "The Advantages of Balloon Frame Exam \#2”, en American Building Construction History, marzo 1, 2007. En: http://rljart.com/blog/wp-content/ balloonframing.pdf (3/09/2018) maderas, de tal manera que se marcaron con un código para erigirse después en el sitio, dando el primer paso para el envío de estas partes a lugares distintos. ${ }^{11}$ El sistema sustituía a las vigas y columnas (post-and-beam) por una estructura de listones más finos y numerosos que son más manejables y pueden clavarse entre sí, una tipología que permitía construir edificios más ligeros y de fácil ensamblaje. ${ }^{12}$

La evolución llevó al surgimiento del platform frame, cuya diferencia fundamental consiste en levantar la estructura planta por planta, interrumpiendo la continuidad de los pilares y resolviendo la dificultad de encontrar piezas de madera de suficiente longitud para abarcar las plantas con una sola pieza de madera. ${ }^{13}$ Los dos sistemas constructivos de marco ligero se expandieron rápidamente debido a una serie de factores: el abundante suministro de madera que había en Estados Unidos, una economía industrial en rápida expansión con clavos de hierro y aserraderos que producían en serie y la fiebre del oro de California, cuando las empresas reclamaron viviendas para la gran cantidad de trabajadores inmigrantes que se asentaron en San Francisco. ${ }^{14}$

Por otra parte, en esta época se incorporó una innovación crucial para las casas prefabricadas, las chapas de hierro corrugado, un elemento que proveía de rapidez constructiva, asequibilidad y eficiencia estructural como material para techos y paredes. La corrosión presentó un problema hasta 1837 cuando las compañías empezaron a galvanizar el metal para protegerlo. El hierro se unió entonces a la exportación de las casas prefabricadas y llegó a infinidad de partes del mundo, ${ }^{15}$ popularizando

11 BOCK, Gordon, "Some Assembly required", Old-House Journal, Vol. 34, No. 1, Jan-Feb-, 78-29, 2006, p. 79. En: https://books.google.com.mx/books?id=i6bghkuQGkACEtprintsec $=$ frontcover\&dq $=0 l d$ House +Journal, + Vol+34Cthl=es\&tsa $=$ XEtved=0ahUKEwjmtd-6oLPdAhUDba0KHTca DpIQ6AEIJzAA\#v = onepage $\mathrm{Eq} \& \mathrm{tf}=$ false (06/04/2018)

12 FLORES SASSO, Virginia, "Impacto y origen de la arquitectura prefabricada de madera en la República Dominincana (Siglo XIX y XX)", Actas Vol. 1.indb, 2017, p. 561. En: https:// www.researchgate.net/publication/323346426_Impacto_y_ origen_de_la_arquitectura_prefabricada_de_madera_en_ Republica_Dominicana_Siglo_XIX-XX (12/03/2018).

13 FLORES SASSO, Virginia, "Impacto y origen de la arquitectura prefabricada de madera en la República Dominincana (Siglo XIX y XX)", opus cit., p.561.

14 COLIN, Davies, The Prefabricated Home, Reaktion Books, London, 2005, pp. 44-47.

15 SMITH, Ryan E., "History of Prefabrication: A Cultural Survey”, en Third International Congress on Construction 
la techumbre industrial que se observa en las edificaciones de las fincas del Soconusco y es el elegido para las casas grandes.

Todas estas innovaciones constructivas se difundían por medio de "tratados, manuales, patrones y catálogos creados por paisajistas, arquitectos, ingenieros y carpinteros, que contenían modelos de edificaciones de todo tipo". ${ }^{16}$ Estas guías prácticas o libros descienden de una larga historia de manuales para la construcción y se utilizaron como un recurso importante para divulgar las nuevas técnicas y detalles constructivos. ${ }^{17}$ En un principio las publicaciones estaban destinadas para los profesionales de la arquitectura privados de la competencia y formación académica para el diseño (albañiles, carpinteros, maestros de obra); incluyendo un número de autodidactas. ${ }^{18}$ Los libros en general, incluian dibujos de plantas, detalles constructivos y perspectivas que garantizaban la buena ejecución de la vivienda. Algunos libros de patrones que se popularizaron en la época fueron los de: Alexander Jackson Davis's, Rural Residences (1837); Andrew Jackson Downing's, Cottage Residences (1842) o Calvert Vaux's, Villas and Cottages (1857). ${ }^{19}$

Más adelante se dio un giro fundamental a los manuales o libros de patrones, abandonando el enfoque meramente pedagógico para incursionar en el mundo publicitario de la venta de los componentes prefabricados de casas domésticas. Los nuevos catálogos ya no ofrecían casas de paneles portátiles, sino por el contrario, se publicitaban edificios recreativos, fundamentalmente vacacionales, con numerosas

History, Brandemburg University of Technology, Cottbus, Alemania, 2009.

16 FLORES SASSO, Virginia, "Impacto y origen de la arquitectura prefabricada de madera en la República Dominincana (Siglo XIX y XX)”, opus cit., p. 561.

17 REIFF, Daniel D., Houses from Books: Treatises, Pattern, Books, and Catalogs in American architecture 1738-1950: A History and Guide, Penn State Press, Pennsylvania, 2010, pp. 395-397.

18 Construir una casa a través de libros no es nuevo en la historia del mundo arquitectónico, probablemente, el primer libro de diseños y dibujos de elementos arquitectónico se remota al famoso libro De Architectura, del ingeniero romano Vitruvio. Sus ideas sentaron las bases para la evolución de los libros de patrones y la remodelación de estilos arquitectónicos y de estética durante los siglos venideros.

19 MORAIS, Caroline; CLARK, Raymond; KRAWCZYK, Robert J. y MALLGRAVE, Harry F., "Catherine Beecher's Home Desings and Enviromental Control Concepts", PLEA 2009, $26^{\text {th }}$ Conference on Passive and Low Energy Architecture, Quebec, Canada, 22-24 June, 2009. compañías por todo el país que las promocionaban (fig. 1). Su gran desarrollo se vio favorecido gracias al ferrocarril y a partir de 1879 , gracias a una legislación postal que redujo los costes de los envíos, momento en que se intensificaron las ventas de componentes prefabricados para las casas. ${ }^{20}$

A partir de 1900, con el auge constructivo, surgieron compañías importantes que acapararon el mercado de las casas por correspondencia: Sears, Roebuck and Company, Aladdin Homes, Gordon-Van Tine Homes o Pacific Ready Cut Homes, entre otras. Una de las más exitosas fue Sears, que se valió de eficientes estrategias de marketing para vender no solo en las grandes ciudades, con asentamientos en expansión en los suburbios; sino también a los clientes rurales, a los que proveía, a través de sus catálogos, de ropa e infinidad de artículos. ${ }^{21} \mathrm{Su}$ éxito se debió a que supo solventar la crisis de viviendas que tuvo Estados Unidos al final de la Revolución Industrial y el fin de la Primera Guerra Mundial, entre 1890 a 1910, cuando se incrementó en un 50\% la población. ${ }^{22}$

En ésta época lo que el cliente recibía eran los planos de la casa elegida (blueprints) para ser construida con materiales locales. Posteriormente los proveedores de casas incrementaron sus ventas ofreciendo planos, materiales y carpintería propia. Por último, además de vender planos y madera, algunas compañías vieron la oportunidad de ganar más dinero incluyendo componentes prefabricados como chimeneas, ventanas, marcos de puertas, herrajes y escaleras. Con estas nuevas innovaciones en la construcción, el avance tecnológico, la producción en masa de componentes de construcción, el crecimiento de la industria maderera y la introducción de catálogos por correo, los consumidores, constructores y las compañias se apoderaron de esta nueva tendencia en la construcción.

\footnotetext{
HUNTER, Rebecca L., Mail-Order Homes, Sear homes and other kit homes, Shire Publications, USA, 2012, p. 1 ${ }^{21}$ COOKE, Amanda y FRIEDMAN, Avi, "Ahead of Their Time: Sear Catalogue Prefabricated Homes”, Journal of Desing History, Vol. 14, No. 1, Oxford, 2001, pp. 5370. En: https://www.jstor.org/stable/3527272?seq=1\# page_scan_tab_contents (09/02/2018)

22 SCHWEITZER, Robert y DAVIS, Michael W. R., America's Favorite Homes: Mail-Order Catalogues as a Guide to Popular Early 20 $0^{\text {th }}$-Century Houses (Great Lakes Books Series), Wayne State University Press, USA, 1990, p. 65.
} 


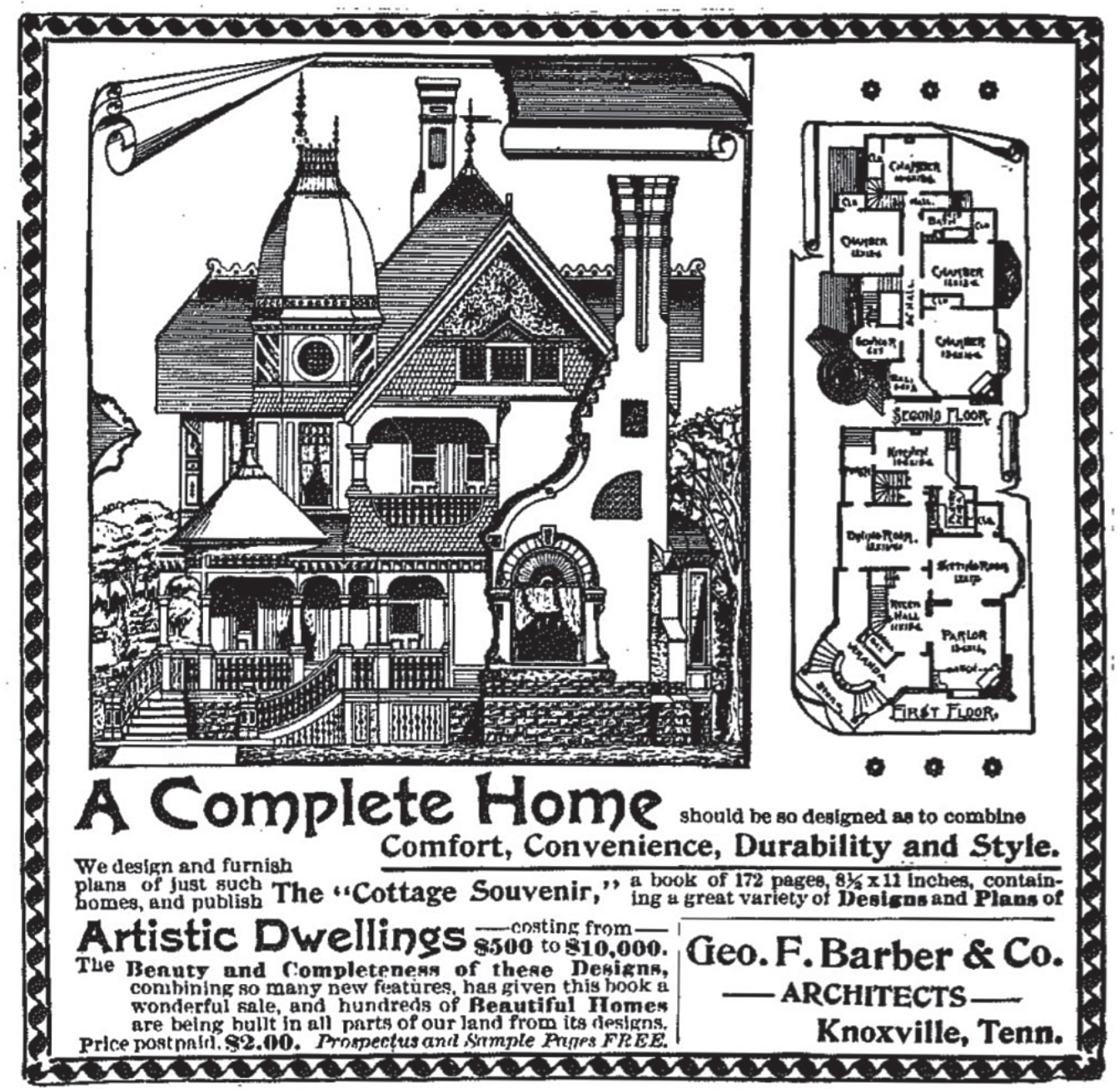

Fig. 1. Anuncio de una casa prefabricada, 1890-1900. Fuente: Leonard de Vries e Ilonka van Amstel, The Wonderful world of American Advertisements 1865-1900, Library of Congress, 1972.

Como se comentó anteriormente, las estrategias de marketing fueron decisivas para la venta de los catálogos, con anuncios en periódicos nacionales o revistas de las ciudades más grandes. La adquisición de la casa la realizaba el comprador eligiendo un modelo de un catálogo de casas que había recibido por correo, previa petición y después de haberla visto anunciada. Otra forma de selección de las casas fue a través de las ferias regionales o nacionales que se celebraron a lo largo del territorio estadounidense, allí las compañías exhibían de manera permanente o itinerante la oferta de casas y los visitantes daban su dirección a las compañías para visitarlos personalmente en su hogar. ${ }^{23}$

HUNTER, Rebecca L., Mail-Order Homes, Sear homes and other kit homes, opus cit., p. 15.
Aunque estos catálogos son los únicos documentos históricos, debemos de ser muy cuidadosos con los datos que ofrecen, ya que son materiales de publicidad, como el correo basura de hoy en día, en el que proliferan las frases engañosas para competir con garantías con los otros. Las compañías afirmaban que el sistema abarataba hasta un 30\% la labor en construcción comparándolo con los métodos tradicionales. Sears por ejemplo, decía que en términos de tiempo tardaba con un modelo de casa 14 días en construirse, a diferencia de los 25 días del método tradicional. Sin embargo, no hay suficientes razones para sustentar las cantidades y a menudo eran parte de las estrategias, como las descripciones insustanciales de las casas, en las que se muestra a mujeres en el interior del hogar y 
poco interesadas en los detalles de la construcción. ${ }^{24}$

En cuanto a los diseños, éstos solían seguir las tendencias arquitectónicas de moda y pocas veces tenían un único estilo, ya que podían modificarse y adaptarse al gusto del cliente, cambiando incluso la planta y los materiales. Dichos recursos fueron, una vez más, una importante estrategia de venta, porque el comprador sentía que podía adquirir una casa personalizada que nadie más tenía. En este sentido, la prefabricación de elementos individuales como ventanas o puertas, además de tener un fın práctico, permitía personalizar la vivienda.

Los catálogos de casas rompieron con la exclusividad, el privilegio e intimidad propia de los proyectos vinculados de modo directo al profesional y es indudable que son una contribución para la actual cultura icónica de masas. ${ }^{25} \mathrm{Su}$ decadencia se produjo a partir de la Segunda Guerra Mundial, cuando el país tuvo una nueva crisis de viviendas y se construyeron un gran número de estructuras prefabricadas para albergar a los veteranos que regresaban. Desafortunadamente se prestó poca atención a la durabilidad de estas casas, desatendiendo su aspecto estético y de calidad en los materiales, incluso cuando se trataba de viviendas permanentes. Ya en 1946 la prefabricación de viviendas en Estados Unidos había desarrollado tan mala reputación, que la percepción continúa hasta nuestros días, por lo que su declive fue imparable.

\section{Las casas prefabricadas del Soconusco}

Con el establecimiento de los primeros inversores y familias germano-guatemaltecas en la región fronteriza del sur de México, la producción de café lavado de exportación se convirtió en el principal negocio del Soconusco a partir de 1890. El súbito incremento de la producción requería un crecimiento igualmente rápido de los procesos industriales y para solventar esta necesidad se adquirieron bienes a través de catálogos impresos que ofrecían

24 HUNTER, Rebecca L., Mail-Order Homes, Sear homes and other kit homes, opus cit., p. 1.

25 ÁlVAREZ QUINTANA, Covadonga, "Publicaciones gráficas de arquitectura doméstica (1800 1925)”, Liño: Revista anual de historia del arte, № 5, Oviedo, 1985, pp. 205-226. muebles, implementos y maquinaria agrícola, así como casas prefabricadas que podían ordenarse por correo. Se creó entonces una élite de empresarios que disponían de capital suficiente para modernizar las fincas e industrializar los procesos productivos del café.

Así se entiende, cómo la fiebre mundial por la industrialización completa tuvo su consecuente impacto en los sistemas constructivos de las fincas del Soconusco, cambiando el paisaje arquitectónico de la región. La adquisición de la casa grande ${ }^{26}$, comprada vía postal y con su sistema constructivo basado en la prefabricación de elementos y posterior ensamblaje en obra, es parte de este fenómeno que se vivió a finales del siglo XIX y principios del XX en la industria del café. El resto de edificaciones de las fincas también se modernizaron y emplearon nuevos materiales de construcción como: el ladrillo, el hormigón, la fundición, el hierro forjado y la madera fundamentalmente. Las nuevas tecnologías, el hierro y el soporte de acero, se destinaron sobre todo para la zona industrial de los beneficios.

Pero además, hay dos aspectos fundamentales para entender la elección de este sistema constructivo para las fincas del Soconusco. El primero de ellos es la pervivencia de una tradición que se venía reproduciendo en la zona guatemalteca: la de residir en una casa prefabricada comprada en el extranjero. A Guatemala llegaron las primeras casas portátiles procedentes de Estados Unidos a mediados del siglo XIX, ${ }^{27}$ realizadas en madera y coincidiendo con aquella industrialización de ciertos elementos de las viviendas producto de la fiebre del oro de California. El segundo motivo se relaciona con la migración de estas poblaciones asentadas en la región fronteriza de ambos países ya que, como se ha señalado, históricamente la ambición por la industrialización completa de la fabricación de edificios surge de la demanda generada por circunstancias

\footnotetext{
26 La casa grande es el término que se utiliza para la vivienda del patrón y la familia del finquero, ocupa un lugar preponderante dentro del espacio habitado de la finca y sus características constructivas la suelen singularizar.

27 ESCOBAR MALDONADO, B. del C. y MORÁlES MARROQUÍN, R., Arquitectura para la producción de café en las fincas de alemanes del Departamento de San Marcos. Análisis y estado de conservación de la casa patronal de la comunidad agraria El Porvenir. Tesis de licenciatura en arquitectura, Universidad San Carlos de Guatemala, Facultad de arquitectura, 2006
} 
inusuales y un terreno particularmente fértil para tales intentos ha sido el de las poblaciones migrantes, con asentamientos aislados en lugar de los asentamientos propiamente establecidos. ${ }^{28}$

Lo anterior esclarece la popularidad de las casas prefabricadas entre los inmigrantes cafetaleros en Guatemala, particularmente los de ascendencia alemana. Este "buen gusto" o "buen estilo" de comprar una casa con elementos prefabricados se trasladó al Soconusco cuando las familias migraron a México a finales del siglo XIX. Se reprodujo entonces la tradición de comprar una casa prefabricada incluso en fechas tardías, entre finqueros sin ningún vínculo con el país vecino y a pesar de la abundancia y calidad de maderas de la región. Cada familia eligió su propio modelo, que llegó a convertirse en símbolo de identidad de la empresa familiar, pero también del éxito y la modernidad.

Respecto a la cronología, la primera referencia documental sobre una casa de estas características se fecha en torno a 1900, procedente de un juicio testamentario y en el que se alude a la edificación como "casa con madera de California" ${ }^{29}$. Podemos deducir entontes que llegaron estas casas a finales del siglo XIX y que para inicios del siglo siguiente las casas estaban asentadas en la región. Debe notarse que se hace un especial énfasis en el tipo de madera como la cualidad más destacable de la edificación, un hecho que devino en la confusión con el estilo, ya que posteriormente se les denominó como "casas de estilo californiano" o "georgiano", confundiendo la procedencia con el estilo arquitectónico.

Ahora bien, es indudable que las casas provenían de la Costa Oeste del país vecino y probablemente fueron embarcadas en el Puerto de San Francisco para su traslado al Soconusco, aunque la falta de documentos aduanales ha dificultado el establecimiento de una línea clara de transporte de estas casas. En este sentido, se tiene que considerar que la aduana federal del Puerto de San Benito, en Tapachula, se consti-

$\overline{28}$ BIGNELL, Marty, "Some Assembly Required: Component and Ensemble in Prefabricated Australian Domestic Construction", opus cit., p. 427.

29 Archivo Judicial Regional del Soconusco (en adelante, AJRS), 1900/58, Juzgado de Primera Instancia del Ramo Civil del Soconusco, Juicio: Sucesión de Manuel Y de la Cerda. tuyó de manera oficial en fechas posteriores a la entrada de las casas. ${ }^{30}$

En cuanto a las últimas construcciones hay más certeza, a finales de la década de 1920 se dejaron de comprar debido al declive de la venta de casas por correo durante la Gran Depresión en Estados Unidos y porque los finqueros comenzaron a hacer uso de maderas locales para las nuevas edificaciones. Hasta ese momento, la madera de las casas prefabricadas procedentes de la Costa Oeste de Estados Unidos fue la sequoia de California o reedwood, una conífera muy abundante en los bosques del norte de California y Oregón y que destaca por su altura y su calidad como material para la construcción. ${ }^{31}$

Respecto al estilo arquitectónico podemos afirmar que no está unificado, de hecho se mezclan diferentes diseños y estilos de cada época, causando una confusión en su identificación. Las casas reproducen los diseños más populares de la Costa Oeste de Estados Unidos y se explica porque los modelos de las compañías eran diferentes de una costa a otra zona. Además, una práctica habitual entre compañías era copiarse los modelos y diferenciarlos únicamente, debido a los impedimentos legales, con modificaciones triviales o difíciles de apreciar.

En los primeros catálogos son frecuentes los diseños vernáculos con un puñado de elementos victorianos, coincidiendo además con las primeras construcciones que llegaron al Soconusco. La simpleza de estas construcciones es evidente, semejantes a las cabañas de los primeros pioneros estadounidenses, conocidas en inglés como log cabin. Posteriormente, el estilo que prevaleció en la Costa Oeste y que coincide con ciertas construcciones de las fincas es el estilo bungalow de California, una vivienda de una sola planta o planta y media, techos bajos, infınidad de ventanas y grandes porches.

Ahora bien, no se pueden identificar las casas del Soconusco exclusivamente por los estilos arquitectónicos y comparándolas con

\footnotetext{
30 Archivo General de Centroamérica y Secretaría de Relaciones Exteriores (en adelante, AGC- SRE), 1909, Exp. 139, Fo. 6, Adquirimientos de un terreno del gobierno federal para la construcción de la aduana de San Benito.

31 Con la fiebre del oro de California madereros y buscadores de oro talaron estos grandes árboles para construir casas con el famoso sistema de armazón simple o balloon frame, uno de los sistemas más populares para las casas prefabricadas de los catálogos.
} 
los catálogos, ya que no son suficientes. Como muchas casas son copias de otras compañias $\mathrm{o}$ arquitectos, los exteriores no corroboran su procedencia. Por el contrario, los indicios que nos pueden hablar de los orígenes de las casas se pueden buscar en los números las vigas, techos, zonas de la pared o peldaños de las escaleras, aunque no suelen estar a la vista. Las anotaciones suelen ser a mano porque solo algunas compañías usaron tinta para estampar a partir de 1930. Las pruebas adicionales para detectar si es un edificio prefabricado se tienen que buscar entonces en elementos estructurales que evidencien la factura del ensamblaje, en los planos originales (blueprints) con el nombre y el número de envío, el número de casa y logo de la compañía, los documentos de correspondencia, la garantía, el manual de instrucciones o lista de partes, la lista de las tasas aduanales del comprador o del vendedor, así como las etiquetas de envío.

Es así como se realizó la búsqueda de las casas prefabricadas del Soconusco y se pueden ejemplificar en este trabajo dos edificios emblemáticos que pertenecen a la familia Giesemann, asentada en la región a partir de 1890. Sus propiedades se han reducido a lo largo de las generaciones, ${ }^{32}$ pero en la actualidad cuentan con una serie de viviendas prefabricadas que ejemplifican claramente el comercio de casas que se realizó en esta zona de México.

\section{Finca Argovia}

La finca Argovia cuenta con tres edificios prefabricados, el primero de ellos es la administración, construida alrededor de 1880, fecha anterior a la adquisición de la familia Giesemann. ${ }^{33}$ El otro edificio se corresponde con la casa del administrador, de cronología incierta pero que suponemos es posterior a 1910. Finalmente, la casa grande de la finca Argovia, la construcción más documentada hasta la fecha y construida en 1905, según inscripción del friso de la fachada. ${ }^{34}$

32 VV. AA., Los empresarios alemanes, el Tercer Reich y la oposición de derecha de Cárdenas (I), CIESAS-Cuadernos de la Casa Chata, México D.F., 1988, pp. 74-75.

33 Esta información fue obtenida a través de una entrevista realizada a señor Bruno Giesemann, propietario de la finca Argovia y entrevistado el 28 de octubre de 2017.

34 Debido a la polilla, la casa grande se remodeló y reconstruyó en el año 1999. En el taller de carpintería de la finca se realizó el machimbrado bajo el sistema de

\section{a) La casa grande}

Es el mejor documentado porque conserva los blueprints o copias del plano original, en donde se indica el nombre de la compañía constructora, el modelo de la casa, además de una serie de instrucciones para su ensamblaje. La construcción fue realizada en 1905 por la compañía Redimade Building Company, una pequeña empresa cuya fábrica se ubicaba en Portland, Oregon, aunque con sucursales también en Los Ángeles y Oakland, California (fig. 2). El pico de ventas se registró alrededor de 1920 y la casa grande de Argovia ejemplifica uno de sus primeros modelos de la compañía que desapareció, como muchas otras, al iniciar la Gran Depresión. ${ }^{35}$

El edificio es una "casa seccional" (sectional house), el cual ha sido escasamente identificado en Estados Unidos debido a que su construcción de paneles livianos lo hizo poco efectivo contra fugas y corrientes; por ello a lo largo de los años la mayoría de casas seccionales se cubrieron con diversos productos para techos y revestimientos, dificultando su identificación. ${ }^{36}$ En Argovia nos encontramos con un ejemplo significativo de casa seccional que cobra relevancia por su escasa identificación en Estados Unidos y por estar ubicada en territorio mexicano.

El modelo de casa es el número 33, una construcción tipo bungalow que cobró fama en el estado de California y que el historiador Tom Hubka denominó como "bungalow porticado". En Argovia sin embargo, vemos una variante local denominada "bungalow de Portland", haciendo referencia a la ciudad de proceden-

gotero, una fórmula que facilita el goteo descendiente de la lluvia. Se mantuvo el cuerpo exterior de la casa y se reconstruyó el interior con maderas locales: huanacaxtle, cedro rojo y primavera. Se realizaron también modificaciones en el cielo, disminuyendo su elevación e introduciendo un mezzanine ventilado y con una estructura diferente. En esa reconstrucción se encontró la firma del constructor de la casa, apellidado Lesher, de origen alemán pero radicado en Guatemala.

35 KENNY, J. (2015). "The Story of One Bungalow \& Portland's Redimade Building Co." Eastmoreland Neighborhood Association News, spring, Portland, 2015. En: http://www.eastmorelandpdx.org/enanewsletter-2/ (02/10/2017).

36 Esta información fue obtenida a través de una entrevista realizada al Mtro. Dale Wolicki, arquitecto y especialista en el estudio de las compañías de casas prefabricadas en Estados Unidos. Entrevistado en diciembre de 2017 a través de medios electrónicos. 


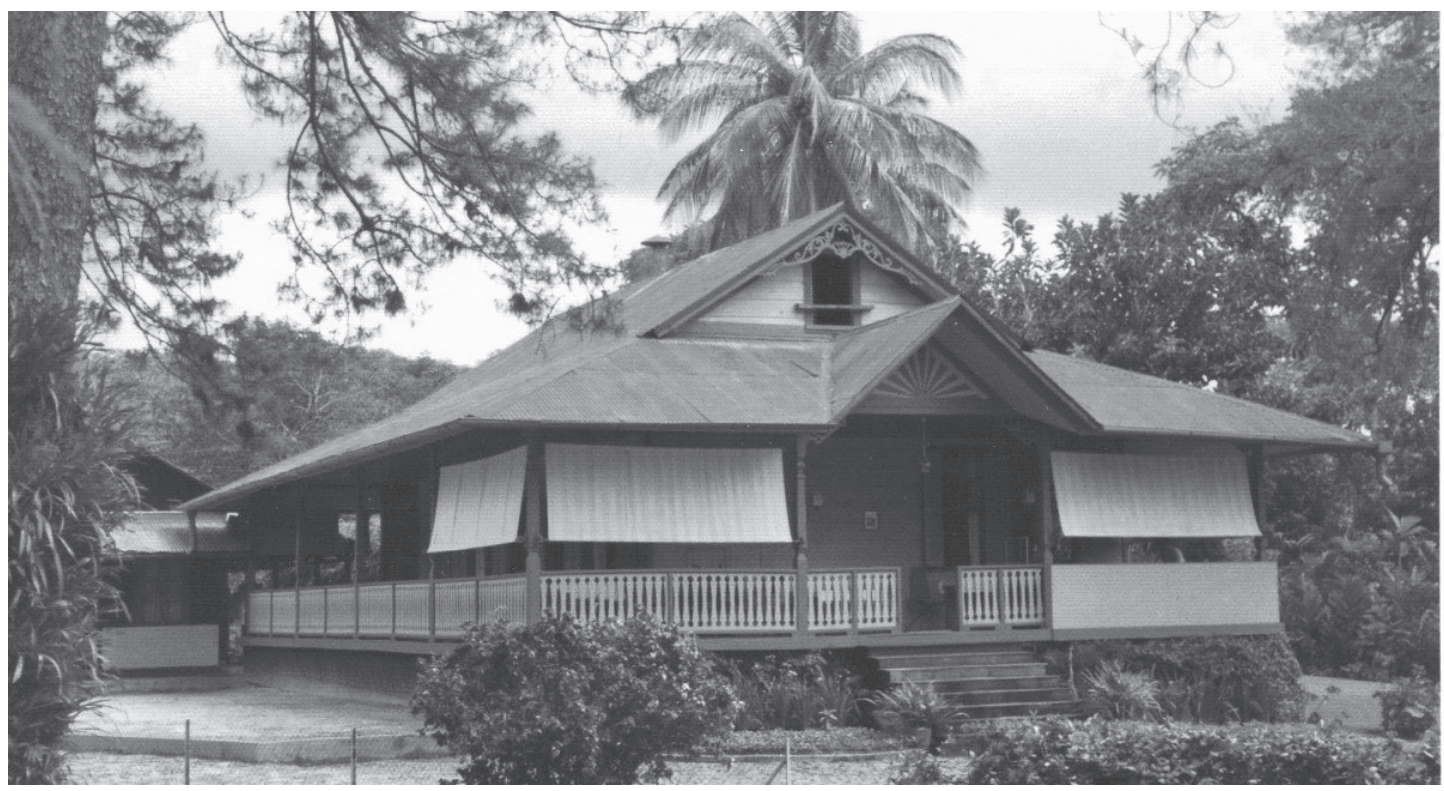

Fig. 2. Casa Grande de la finca Argovia, ca. 1955. Archivo familiar de la familia Giesemann.

cia. ${ }^{37} \mathrm{El}$ estilo arquitectónico se volvió popular entre 1900 y 1920, "personificando la esencia del estilo de vida y sueño de California, donde los estadounidenses podían vivir en su pequeña casa de madera rodeada de palmeras y plantaciones tropicales". ${ }^{38}$ Efectivamente, es un diseño práctico y económico que encaja a la perfección con este modo de vida en el campo de la familia Giesemann.

En términos de estructura, es un cuerpo de un solo nivel cuyo centro se encuentra rodeado de un porche abierto que envuelve la casa. Este espacio, además de concebirse como un elemento estético, cumple con una función básica: dotar a la casa de una ventilación constante en un clima caluroso y húmedo, permitiendo la ventilación cruzada por varios frentes de la casa, pero también protegiendo el muro perimetral de la lluvia y el sol que invaden la vivienda y que erosionan la madera. La arquitectura se adapta a las condiciones impuestas por la naturaleza ya permite la entrada de aire fresco. La cubierta protege de las intensas lluvias

Ibídem

38 GREENE MALVASI, Meg, The Kit House in Southeastern Virginia. Architectural Documentation of Kit Houses Manufactured by Sears, Roebuck and Company Located within the Cities of Chesapeake, Norfolk, Portsmouth, Suffolk, and Virginia Beach, Virginia, William and Mary Center for Archeological Research, Virginia, 2006, p. 34. En: https://www.dhr.virginia.gov/pdf_files/ SpecialCollections/VA68_Sears_Kit_Houses_2006_ Report.pdf (24/08/2018) y la construcción de madera brinda seguridad frente a los movimientos telúricos. La abertura de un espacio al exterior invita a un mayor contacto con la naturaleza, pero también a la vigilancia y control. El porche es por lo tanto un elemento fundamental de la vida en la casa grande, ya que el mayor tiempo de la vida cotidiana se realiza en ese espacio. La cocina se ubica al exterior de la casa y se conecta, como muchas otras edificaciones y por cuestiones de seguridad, al edificio principal por medio de un corredor.

En el interior de la vivienda se distribuyen las habitaciones o estancias familiares separadas por muros de madera. La ventilación interior se consigue gracias a las ventanas distribuidas por toda la casa, pero también gracias al cielo interior, que en su versión original era de mayor altura que el actual. Además la vivienda no se ubica a ras del suelo, sino sobre una estructura vigas horizontales que posibilita la ventilación desde abajo. Esta elevación de las viviendas es habitual en las casas prefabricadas del Soconusco, como una manera de proteger la madera frente a la humedad del suelo.

El techo inclinado a dos aguas posibilita el desagüe y su cubierta se realiza con la característica lámina de zinc roja, elemento económico, de fácil disposición y perdurable. En el hastial de la estructura original se pueden observar detalles de estilo victoriano que se asemejan a los realizados por la compañía American Portable House Company, con sede en Seattle, Washing- 


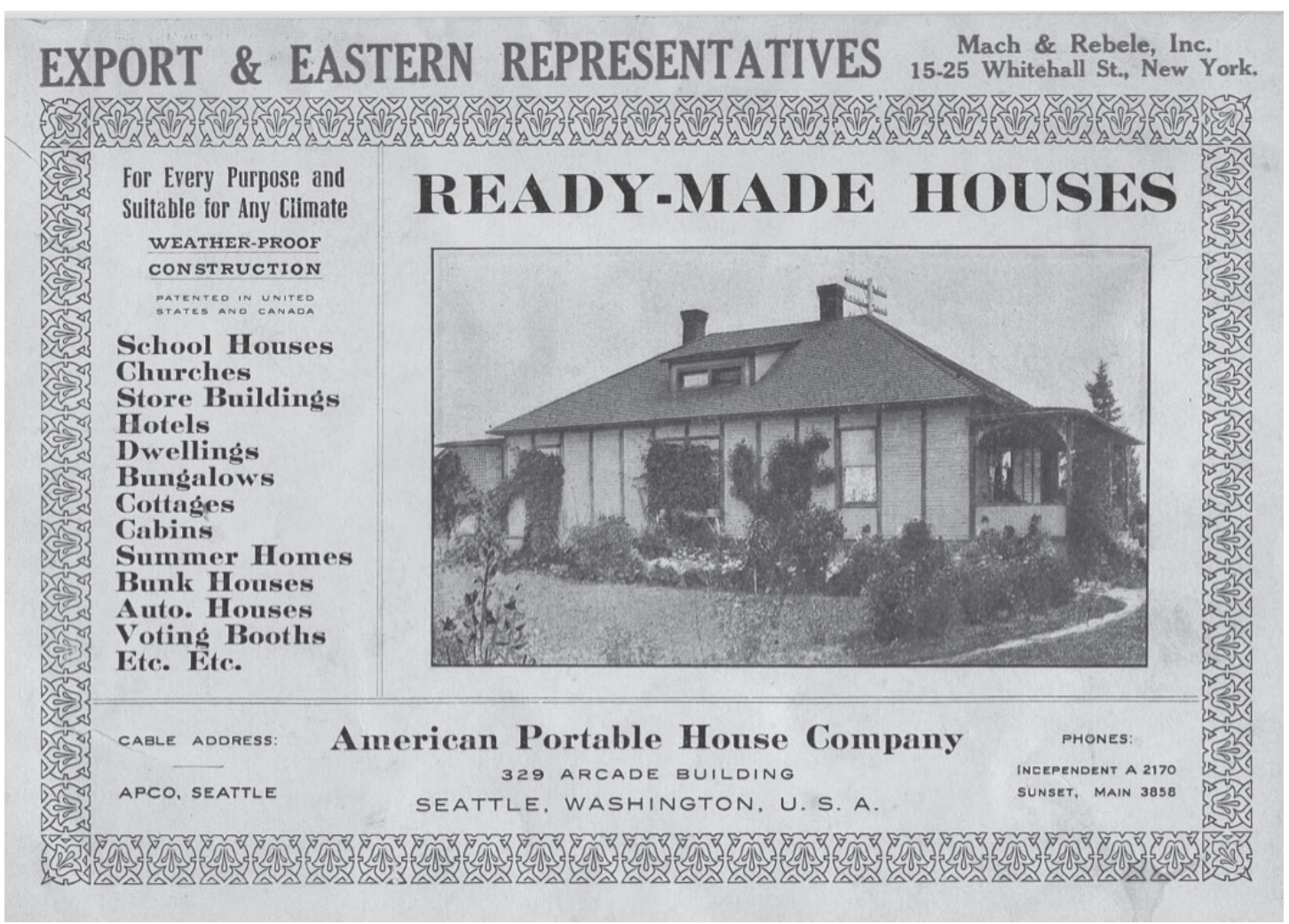

Fig. 3. Anuncio de la Compañía American Portable House Company, 1910. Archivo personal del arquitecto Dale Wolicki.

ton. Una empresa que operó aproximadamente entre 1896 y 1922 y que fue la mayor fábrica de sectional-portable buildings ${ }^{39}$ de la Costa Oeste de Estados Unidos de América. La empresa se especializó en la construcción de escuelas, pero también ofrecía iglesias, clínicas y grandes casas de seis habitaciones. Se tiene conocimiento de envíos a ultramar y se han identificado construcciones en Hawai y Filipinas, por lo que es probable que hiciera envíos a México (fig. 3). Todos estos datos nos hablan de dos compañías implicadas en la construcción de la casa, un hecho que no puede pasar desapercibido y que sienta precedente de cómo se realizaron los pedidos de las casas del Soconusco.

\section{Finca La Violeta}

La casa grande de la finca La Violeta sigue el mismo estilo de bulgalow o cottage de

39 Se tiene que tener en cuenta que las "casas portátiles" son las mismas que las "casas seccionales". La oficina de Patentes de Estados Unidos definió algunas estas estructuras prefabricadas como "portáltiles", pero algunos fabricantes prefirieron llamarlas "seccionales". verano que puede ser apreciable en muchos de los catálogos de 1920, aunque con sus respectivas particularidades. La construcción se fecha entre 1920 y 1925, según fotografías y referencias de su actual propietario. No poseemos documentación complementaria del edificio, pero ciertos elementos estructurales evidencian el ensamblaje de las piezas, además del estilo arquitectónico. Respecto al sistema constructivo, probablemente estemos ante un ejemplo de casa pre-cortada (pre-cut home), el sistema comúnmente utilizado a principios del siglo XX. ${ }^{40}$

Visualmente es una estructura simétrica, un cuerpo cuadrangular de planta y media ${ }^{41} \mathrm{cu}-$ bierto a cuatro aguas (fig. 4). La línea del tejado se rompe visualmente por las cuatro buhardillas que organizan el espacio superior en una especie de cruz griega, coincidiendo con tres

40 A partir de 1906 el sistema constructivo para las casas prefabricadas fue el mismo, aunque cada compañía utilizaba el término indistintamente: pre-cut homes, kit houses, mill-cut houses, ready-cut houses, mail order homes y catalog homes.

${ }_{41}$ Es habitual que en los catálogos se considere como media planta al espacio entre buhardillas. 


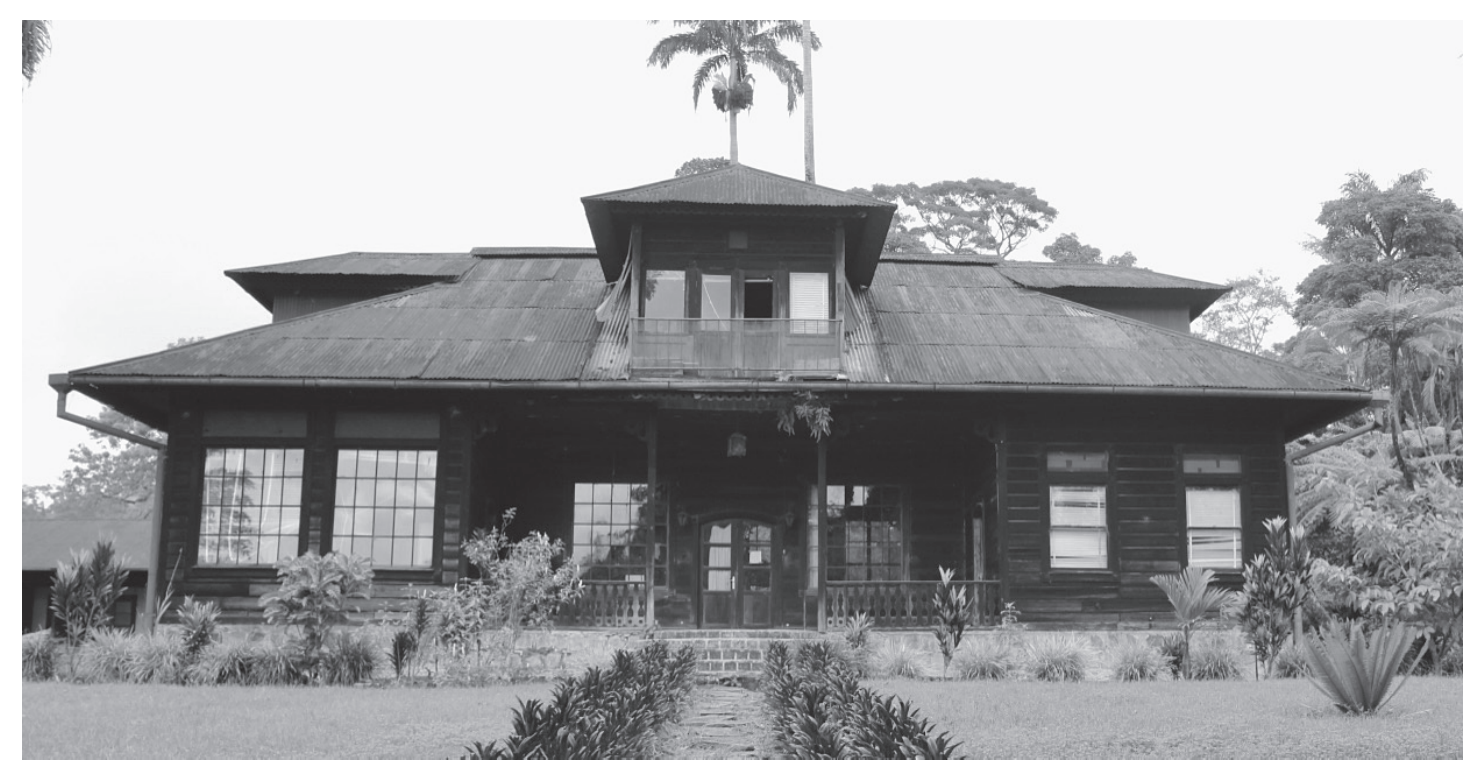

Fig. 4. Casa grande de la finca La Violeta, 2017. Fotografía de la autora.

dormitorios y una estancia central. La entrada principal a la casa se realiza por medio de una terraza ubicada en el centro de la fachada del edificio, aunque lateralmente también se dispusieron accesos de menor envergadura. En la cara posterior del edificio se encuentra otra terraza que permite el acceso a la escalera que conduce al nivel superior y al único baño de la vivienda.

En la planta principal se organizan seis espacios: la sala de costura, dos dormitorios, un salón principal, una sala de usos múltiples y un comedor. Es una casa de grandes dimensiones construida con madera de redwood de California y cubierta con lámina de zinc roja. A pesar de su antigüedad conserva muchos de los elementos originales que fueron adquiridos ex profeso para la casa: las puertas, las ventanas con sus respectivas molduras decorativas o los lavabos personales de los dormitorios de la planta baja, un elemento privilegiado para la época (fig. 5). El suelo de toda la vivienda es de madera, excepto en la sala de usos múltiples y el comedor, cubierto con baldosas de cemento hidráulico realizadas por artesanos en la propia finca. Grandes ventanales de veinte paneles permiten la entrada de luz en los espacios comunes y sus proporciones arrojan pistas sobre su construcción. ${ }^{42}$ En el otro extremo del edificio y coincidiendo con los

42 Heritage House Notes at Built Heritage Research Guide, PEI Museum y Heritage Foundation, Charlottetown, dormitorios, se ubican las ventanas de guillotina de menores dimensiones y de panel sobre panel. La cocina se encuentra en el exterior de la casa y se conecta por medio de un corredor abierto.

\section{Conclusiones}

Las fincas constituyen un exponente significativo de un sistema agroindustrial vinculado a procesos socio-culturales de gran incidencia en la memoria hostórica de esta zona fronteriza de México. Las casas grandes exhiben el legado cultural que aportó la inmigración alemana a la región. Estas viviendas contienen valores, materiales e inmateriales, relacionados con la cultura cafetalera y han intervenido en la configuración de la identidad cultural soconusqueña, principalmente en las zonas rurales donde las construcciones evidencian, a través de la adaptación, una similitud de componenentes arquitectónicos. Las casas que aquí se han expuesto son una pequeña muestra del rico patrimonio arquitectónico por estudiar.

Canadá, 2010. En: http://www.gov.pe.ca/photos/original/ HeritageHouseNo.pdf (25/06/2018) 


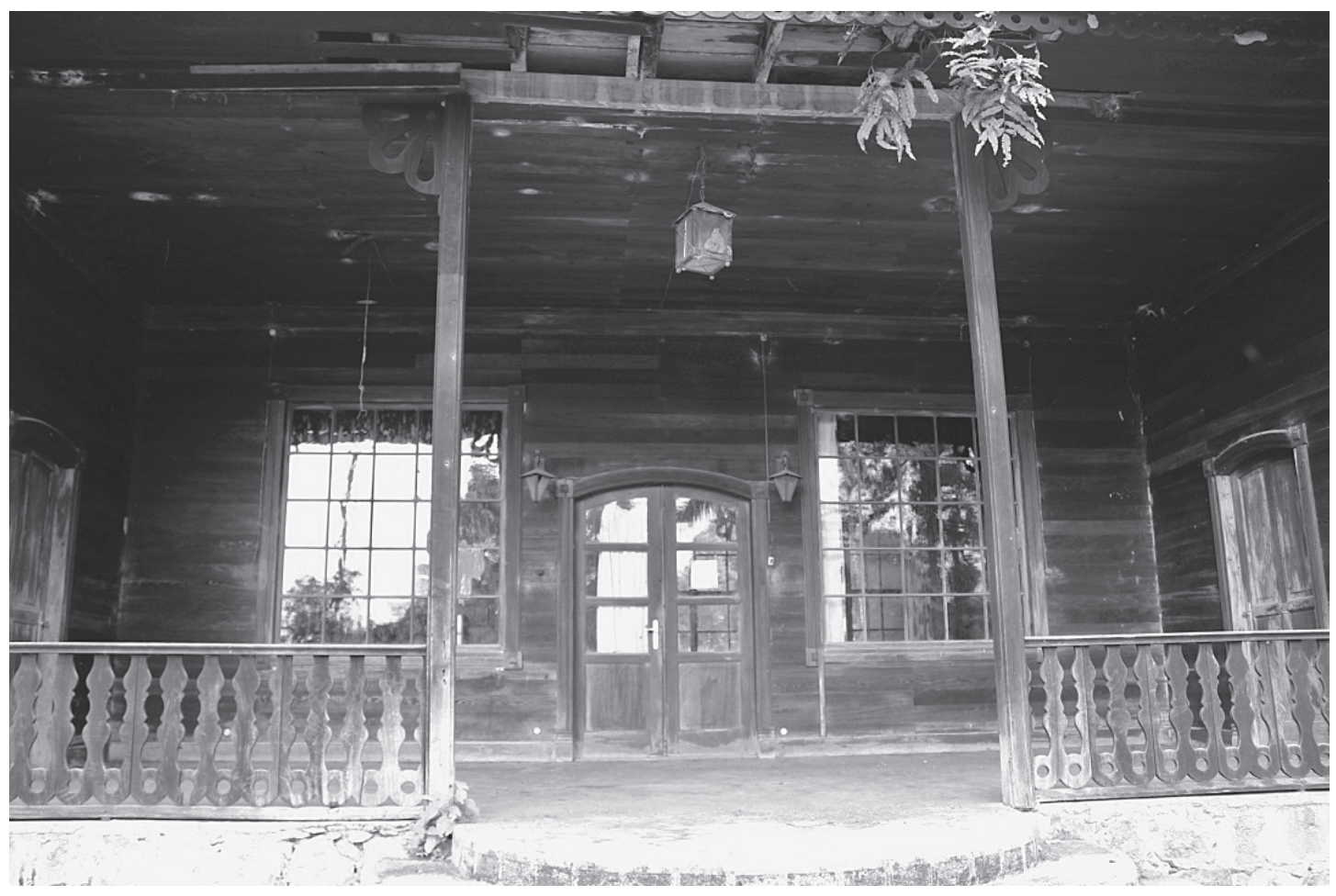

Fig. 5. Vista lateral de la casa grande de la finca La Violeta, 1952. Archivo personal de la familia Gisemann. 